\title{
Estética política y teoría de la literatura: un diálogo abierto
}

\section{Political Aesthetics and Theory of Literature: an Open Dialogue}

\author{
Azucena GonZÁlEz BlANCO \\ Universidad de Granada \\ azucena@ugr.es
}

Recibido: 10/07/2013

Aceptado: 30/05/2014

\section{Resumen}

La teoría de la literatura se encuentra hoy ante el reto de definir un nuevo espacio para el estudio de la literatura en toda su plurivocidad. Por este motivo, se propone defender la apertura de la teoría de la literatura a las propuestas realizadas desde algunas posturas del giro político en la estética y la teoría. Nuestra hipótesis es que el diálogo entre estas disciplinas ayudaría a comprender y a responder-endiálogo a una cuestión que ha recorrido, desde la entrada en juego de las vanguardias históricas, la estética y la teoría literaria contemporáneas, a saber: la dialéctica entre autonomía literaria y la literatura en la comunidad.

Palabras clave: Estética política, teoría de la literatura, comunidad, autonomía, J.-L Nancy, C. Menke, J. Rancière.

\begin{abstract}
Theory of literature is nowadays confronted with the challenge of defining a new space for studying literature in all its plurivocity. Therefore, I propose to defend the opening of/from theory of literature toward the realized proposals from some propositions of the political turn in aesthetics and theory. My hypothesis is that the dialogue between these disciplines will help to understand and respond a recurring question within the dialogue, from the introduction of the historical avant-garde, aesthetics, and contemporary literary theory, to knowledge: dialectics between literary autonomy and literature in the community.

Keywords: Political aesthetics, theory of literature, community, autonomy, J.-L Nancy, C. Menke, J. Rancière.
\end{abstract}




\section{Hacia (de vuelta a) la teoría (de la literatura)}

El término teoría de la literatura ha venido perdiendo vigor en los últimos veinte años. Así lo han señalado recientemente, entre otros, los teóricos de la literatura J. Culler en The literary in theory ${ }^{1}$ y más recientemente en "Afterword: theory now and again" , o T. Eagleton en El acontecimiento de la literatura ${ }^{3}$, para quien la teoría de la literatura habría quedado arrinconada por un cuarteto de cuestiones: el poscolonialismo, la multiculturalidad, la sexualidad y los estudios culturales ${ }^{4}$. Este envejecimiento del término teoría de la literatura habría venido acompañado, sin embargo, de un aumento del uso del término teoría. Esta falsa abreviatura vendría a definirse, en términos de J. Culler, como una mezcla de filosofía, psicoanálisis, lingüística, estética, poética y pensamiento político y social:

"Theory as a genre of works began, I have argued elsewhere, as a name for a mixture of philosophy, psychoanalysis, linguistics, aesthetics, poetics, and political and social thought. A text became theory when it was picked up by people in other fields because its account of some phenomenon of general interest seemed promising and productive" 5 .

Esta situación ha supuesto una convivencia de los estudios literarios con los estudios culturales. Y, por lo tanto, una apertura de los estudios literarios a otros valores que no son los exclusivamente literarios. La contradicción, sin embargo, como han señalado estos autores en sus obras citadas, es que finalmente se ha producido un retroceso en los trabajos teóricos que atienden al estudio de las obras literarias en su autonomía. En palabras de J. Culler, en The literary in theory: "El eclipse de la literatura en la teoría es un fenómeno muy reciente. En los primeros días de la teoría lo era de la literatura"6. Por este motivo, J. Culler propone recuperar los trabajos formalistas puesto que el estudio de la literatura muestra también la transformación histórica. Es necesario, dice J. Culler, el estudio de la obra literaria, de sus propios sistemas, para comprender los modos en que las obras individuales subvierten las convenciones. Es necesario el estudio de las condiciones formales de las obras literarias, y no sólo de sus temas. De modo que se pueda estudiar cómo las condiciones formales se enfrentan críticamente con las instituciones de poder ${ }^{7}$.

\footnotetext{
${ }^{1}$ J. Culler, The literary in theory, Standford, Standford University Press, 2007.

${ }^{2}$ G. Farred and M. Hardt (eds.), Theory now, en The South Atlantic Quarterly, 110:1, Invierno 2011, pp. 223-230.

${ }^{3}$ T. Eagleton, El acontecimiento de la literatura, Madrid, Ediciones Península, 2013.

${ }^{4}$ Ibidem, p. 2.

${ }_{6}^{5}$ J. Culler, op .cit., p. 2.

${ }^{6}$ Ibidem, p. 3.

${ }^{7}$ Ibidem, p. 11.
} 
En este mismo sentido, T. Eagleton señalaba en ¿Cómo se lee un poema $?^{8}$ (2007), que había sentido la necesidad de una obra con un título tan próximo a la crítica literaria, porque en sus clases, los alumnos ya no eran capaces de realizar un trabajo de crítica literaria, formación necesaria para atender al análisis formal de las obras. Si bien, otros autores del siglo pasado, habían sido capaces de analizar la obra literaria en toda su plurivocidad, tales son los casos, dice T. Eagleton, de Bajtín, Barthes, Derrida, Kristeva o Foucault ${ }^{9}$.

Este tipo de obras, serían consecuencia de la denuncia del desplazamiento que se ha producido a la cultura que habría obviado la forma literaria. Y ello porque, como afirma J. Culler, el estudio de la forma se habría vuelto a denunciar como una negación de la sociedad a la que pertenece.

Del mismo modo, señala Massimo Fusillo la necesidad de una estética comparada en Estética de la literatura ${ }^{10}$. Según Fusillo, en las últimas décadas del siglo XX, la Estética y la literatura parecían abocadas a una inevitable decadencia. Debido, por una parte, a la denuncia del fracaso de la utopía modernista del arte como revolución, de mano del arte postmoderno, y por otra parte, por la banalización del concepto de gusto subjetivo que se disolvía definitivamente en una cultura de masas y plural. La propuesta de Fusillo, no obstante, es la de replantear la historización de la estética y de su pluralidad cultural:

"la cual considera los juicios estéticos como fenómenos fuertemente condicionados por presupuestos ideológicos (en continuidad, por tanto, con los Cultural Studies); pero que entiende, en cualquier caso, la actividad estética como la respuesta a unas necesidades cognitivas primarias: tanto más importante e insustituible cuanto más nos empuja a salir de nuestro contexto" 11 .

Este debate sobre la actualidad de la teoría de la literatura tiene sus raíces, no obstante, en una problemática mucho más antigua: la dicotomía entre la negación de lo social (el juicio estético desinteresado) y la implicación directa con las grandes ideologías y las utopías políticas, la estética, la teoría y la reflexión sobre la literatura, han recorrido un largo camino.

Nuestra propuesta es retomar la relación entre la estética política y la teoría de la literatura desde una perspectiva horizontal, sin establecer una jerarquía disciplinaria que está en la base de las últimas propuestas de la estética literaria y sin aspiraciones de reificación humanista. Es decir, en términos de R. Rorty, no como

\footnotetext{
${ }^{8}$ T. Eagleton, Cómo se lee un poema, Madrid, Akal, 2010.

${ }^{9}$ Ibidem, pp. 9-11.

${ }^{10}$ M. Fusillo, Estética de la literatura, Madrid, Visor, 2012, pp. 11-23.

${ }^{11}$ Ibidem, p. 13.
} 
recuperación de una verdad que sólo es posible vislumbrar desde la filosofía del arte ${ }^{12}$. La estética y teoría de la literatura encuentran hoy un espacio fronterizo en el que pueden aproximarse desde perspectivas diferentes a problemas constantes del pensamiento, el texto literario, la filosofía y la política. Este diálogo podría ayudar a superar las dificultades entre formalistas y teóricos de la cultura, pues, permitiría establecer una vía de desarrollo de perspectivas no reduccionistas en el estudio de esta ya clásica dicotomía. Y por otra parte, se propondría como un acceso privilegiado al estudio del texto literario desde una vía interdisciplinar que no obvia su doble naturaleza, autónoma y política.

Consideramos que es la recuperación de la dimensión estético-política del texto que se está realizando en los últimos años desde la estética, la que hace posible que los estudios teóricos sobre literatura puedan plantearse de forma renovada la pregunta por la literatura en el espacio social (siguiendo, entre otros, a J. Rancière ${ }^{13}$ ), o el espacio de la comunidad (en el caso de J-L. Nancy ${ }^{14}$ ), sin perder la autonomía que le otorgaron las reflexiones formalistas (como reivindica C. Menke ${ }^{15}$ ).

\section{Precedentes}

El concepto moderno de literatura data de finales del siglo XVIII y principios del XIX, y es contemporáneo de la definición del arte que Kant expone en su Crítica del juicio (1790) como una finalidad sin fin, es decir, autónomo. Como expone José María Cuesta Abad, la teoría moderna de la literatura surgió del encuentro no siempre pacífico, entre la filosofía, la estética y la literatura bajo la influencia dominante de los programas idealistas y románticos ${ }^{16}$. Pero no va a ser hasta los estudios que se reali-

\footnotetext{
${ }^{12}$ R. Rorty, Contingency, irony and solidarity, Nueva York, Cambridge University Press, 1989.

${ }^{13}$ A este respecto, pueden consultarse, entre otras, las obras del autor: La parole muette. Essai sur les contradictions de la littérature, París, Hachette, 1998; Malaise dans l'esthétique, París, Galilèe, 2004; Politique de la littérature, París, Galilée, 2007. (Trad. Política de la literatura, Libros del Zorzal, 2011).

${ }^{14}$ A este respecto, pueden consultarse, entre otras, las obras: J.-L. Nancy y P. Lacoue- Labarthe, L'absolu littéraire. Théorie de la littérature du romantisme allemand, París Seuil, 1978; J. -L. Nancy, La communauté désouvrée, Christian Bourgois (ed.), París, 1999. (Trad. La comunidad desobrada, Madrid, Arena Libros, 2001); J. -L. Nancy, Les Muses, I y II, París Galilée, 2001; J. L. Nancy, La création du monde ou la mondialisation, París, Galilée, 2002.

${ }^{15}$ A este respecto, consúltense las obras del autor: Aesthetics of Equality, Documenta, Kassel, 2011; La actualidad de la tragedia. Ensayo sobre juicio y representación, Madrid, Visor, 2008; Kraft. Ein Grundbegriff ästhetischer Anthropologie, Frankfurt am Main, Suhrkamp Verlag, 2008; Die Souveränität der Kunst: Ästhetische Erfahrung nach Adorno und Derrida, Frankfurt am Main, Suhrkamp Verlag, 1991.

${ }^{16} \mathrm{~J}$. M. Cuesta Abad, La transparencia informe. Filosofía y literatura de Schiller a Nietzsche, Madrid, Abada, 2010.
} 
zan en el ámbito del formalismo ruso de principios del siglo XX, cuando se desarrolle un concepto verdaderamente autónomo de literatura. En estos momentos, la literatura se separa de la estética fundada por Baumgarten y Kant. Se separa, entonces, la Poética, precedente clásico de la teoría de la literatura de la estética, la cual desde el siglo XVIII había integrado a la Poética.

Este nuevo concepto de literatura va a fundamentar su recién adquirida independencia en la naturaleza verbal del texto literario. Es decir, este concepto de literatura nace asociado a las tendencias lingüísticas y positivistas que se desenvuelven en la época. Ello conlleva el olvido de los otros aspectos no medibles desde una perspectiva cientificista. Consecuentemente, se estigmatizan y rechazan en la teoría de la literatura conceptos largamente aceptados como el de "experiencia", de notables connotaciones estéticas (experiencia estética) o el de sujeto del arte (por el recuerdo a la crítica biográfica).

Pero en los años sesenta del siglo XX, las reflexiones teóricas y estéticas sobre el arte y la literatura, comienzan a dirigir su mirada a las relaciones con el espacio social y político: los trabajos de P. Bourdieu, A. Badiou, M. Foucault o F. Jameson, son ejemplos de esta tendencia en sus diferentes enfoques. Si bien, a finales del siglo pasado se produce el denominado giro político en gran parte de las disciplinas humanistas, estableciéndose un diálogo con lo social ya en el espacio de la globalización: un nuevo concepto de mundo se comienza a leer desde conceptos teóricos y comparatistas de la literatura como el de "transdisciplinariedad", propuesto por el teórico alemán de la literatura O. Ette ${ }^{17}$, o el de "planetariedad", que Gayatry Spivak proponía en Death of a discipline ${ }^{18}$. La literatura se plantea de nuevo su lugar en el mundo, y ello se muestra en una abundancia de bibliografía que reflexiona sobre la literatura mundial, y un auge de los estudios comparatistas.

Considero, no obstante, que uno de los precedentes fundamentales para esta propuesta dialogante entre estética y teoría de la literatura como vía de superación de la dialéctica entre la autonomía de la obra de arte y la proyección política de la misma, lo encontramos en las propuestas de M. Foucault, que definía la literatura como un espacio de crítica del lenguaje que recubre todas las instituciones y el poder. Ello porque la afirmación sobre la literatura de que toda forma implica un contenido, fue asumida por las escrituras de filósofos como Jacques Derrida, Gilles Deleuze o Michel Foucault, e hizo que las escrituras de estos filósofos fueran sólo pensables desde la perspectiva de una escritura filosófica que asume la potencia creativa y crítica del lenguaje literario.

Especialmente interesante al respecto, son los últimos trabajos de M. Foucault, que rechazando las posturas nihilistas del texto literario que proponía Paul de Man,

${ }^{17}$ O. Ette, La literatura en movimiento, Madrid, CSIC, 2008.

${ }^{18}$ G. Spivak, Death of a discipline, New York, Columbia University Press, 2003. 
abogaba ya por un texto literario con una potencialidad performativa, que reside en la radical unión de forma/contenido en el texto literario y en su estrategias textuales o retóricas. Michel Foucault reconocía la naturaleza tropológica de todo lenguaje. En "Débat sur le roman", afirma el autor: "Puede ser que el lenguaje del mundo sea una metáfora" 19 . Pero, al contrario que para el teórico de la Escuela de Yale, esta paradoja múltiple del lenguaje, que es a un tiempo constatativo y performativo, formal y represivo, le otorga precisamente una autonomía que no nos permite dominarlo por completo y en ello reside su fuerza creativa y su potencia crítica.

Para Foucault la textualidad, la escritura, es un "campo de batalla" 20 en el que se definen, por un lado, las implicaciones epistemológicas -qué y cómo podemos conocera la vez que éticas -cómo podemos enfrentarnos a los mecanismos de sometimiento del discurso de la historiografía y de las instituciones. Lenguaje como lucha que, por otro lado, asume la materialidad que lo conforma, esto es, el azar del lenguaje diciendo lenguaje (ontología del lenguaje), materialidad que hace imposible ya la relación de transparencia objeto-sujeto. No obstante, esta materialidad no es tratada como un obstáculo ni ambas naturalezas -lenguaje como lucha y lenguaje vuelto sobre sí mismo- como la condición paralizadora del lenguaje. Sino que muy al contrario, son la base sobre la que el discurso "activo" de Foucault fundamenta. En este sentido, la contradicción de la escritura abre, en el umbral de ambas condiciones, una plurivocidad del ser del lenguaje próximo a los términos en los que describe el W. Desmond con respecto al ser:

"Plurivocity of being, prefiguring the dialectical and metaxological senses. Univocity gains its great power from its abstraction from plurivocity, but these other voices come back. As a result, the meaning of univocity itself must be interpreted with more finesse. Plurivocity is the way into and out of contradiction. Contradiction drives us beyond univocity towards plurivocity, and a different communivocity"21.

Foucault define y asume en su propia escritura la naturaleza paradójica del lenguaje, crítica del principio de no-contradicción que se encuentra en la base del pensamiento racional occidental. Es por tanto desde un lenguaje tropológico desde donde podemos comprender conceptos tan fundamentales como el concepto mismo de poder como un concepto retórico-paradójico, que a la reconciliación sintética opone la prolongación de la lucha sin resultado, de lo inacabado ${ }^{22}$.

\footnotetext{
${ }^{19}$ M. Foucault, Dits et écrits (1994), vol. I, París, Galilée, 2003. p. 396.

${ }^{20}$ M. Foucault, El orden del discurso, Barcelona, Tusquets, 1973.

${ }^{21}$ W. Desmond, Being and Between, New York, State University of New York, 1995, p. 83.

${ }^{22}$ Para el desarrollo de estas cuestiones, puede consultarse mi trabajo: "La filosofía y su doble", en J. de la Higuera (ed.), La filosofía y su otro, Granada, EUG, 2013, pp. 215-233.
} 


\title{
3. Literatura y comunidad
}

Finalmente, quiero dedicar un breve apartado a algunas propuestas contemporáneas que han mostrado resultados en este debate sobre la autonomía de la obra literaria en el espacio social, y particularmente, con respecto a la reflexión sobre la literatura y la comunidad. Mostraré algunos de los conceptos que han venido proponiendo autores que se encuentran precisamente en este espacio intermedio entre la teoría y la estética de la literatura, entre la teoría del texto y política, como son R. Rorty, J.-L. Nancy, C. Menke y J. Rancière.

Por su parte, R. Rorty en Contingencia, ironía y solidaridad ${ }^{23}$ apuntaba que si hoy día las cuestiones de fines frente a medios, esto es, las cuestiones de sentido de la propia vida y de la comunidad, son cuestiones de arte y política, es porque se habría aceptado que la verdad se crea del mismo modo que el arte es creativo y no mimético. Y ello porque a finales del siglo veinte, habrían confluido dos corrientes de pensamiento: la que considera que la verdad no se descubre, se crea, y la Romántica que consideraba que el arte no era imitación, sino creación del artista. Los poetas reclamaban para el arte el lugar que en la cultura tradicionalmente habían reclamado para la ciencia. Si bien, ahora, estas dos tendencias habrían aunado fuerzas alcanzando la hegemonía cultural.

\begin{abstract}
"Ahora, estas dos tendencias han aunado fuerzas y han alcanzado la hegemonía cultural. Para la mayor parte de los intelectuales contemporáneos, las cuestiones referentes a fines frente a medios -las cuestiones acerca del modo de dar sentido a la propia vida y a la propia comunidad- son cuestiones de arte o de política, o de ambas cosas, antes que cuestiones de religión, de filosofía o de ciencia"24.
\end{abstract}

Ello habría producido una escisión dentro de la filosofía misma: los que del lado de la ciencia y la ilustración, piensan que la verdad se encuentra no se hace, que la expresión "hacer la verdad" es sólo una expresión metafórica. Así, los filósofos que consideran que la ciencia no nos enseña cómo vivir en el mundo, han llegado a la conclusión de que la ciencia no es más que la sirvienta de la tecnología (razón instrumental). Estos filósofos se habrían alineado, según Rorty, con los utopistas políticos y con los artistas innovadores, y concluyeron que "el mundo no habla, sólo nosotros lo hacemos". De ahí que:

"El cambio de lenguajes y de otras prácticas sociales pueden producir seres humanos de una especie que antes nunca habían existido (...). El método consiste en volver a describir muchas cosas de una manera nueva hasta que se logra crear una pauta de conducta

\footnotetext{
${ }^{23}$ R. Rorty, Contingencia, ironía y solidaridad, Barcelona, Paidós, 2009.

${ }^{24}$ Ibidem, p. 23.
} 
lingüística que la generación en ciernes se siente tentada de adoptar, haciéndoles así buscar nuevas formas de conducta no lingüística. El lenguaje ya no es un medio"25.

El lenguaje adquiere, como para M. Foucault, un poder performativo propio del lenguaje literario, de transformación, "La transformación de lo uno en lo otro", en términos de J. Rancière. De este modo la autonomía de la literatura no se vería puesta en entredicho, si bien, sus rasgos formales serían susceptibles de ser asimilados por la escritura filosófica que sería del mismo modo retórica y performativa (autoimplicación del lenguaje). Según algunas posturas contemporáneas, esta derivación del lenguaje filosófico en lenguaje estratégico-retórico, habría dado lugar no ya al resguardo de la autonomía de la literatura (y del arte), sino más bien a una "soberanía del arte" en términos de C. Menke, en La soberanía del arte. La experiencia estética según Adorno y Derrida ${ }^{26}$.

Esta idea abierta sobre el lenguaje literario es retomada por una figura eslabón entre los dos momentos de reflexión que describimos. Para J. Rancière, la literatura, a diferencia de otras artes, "ha sido el laboratorio en el que se experimentaban las formas de descripción y de interpretación de la experiencia”. Es decir, existe un vínculo inevitable, a través del lenguaje, entre literatura y comunidad. La literatura proporciona modelos y procesos democráticos de la escritura y nos permitiría leer en clave "democrática" las formas narrativas de autores calificados por algunos como apolíticos, como Flaubert, Mallarmé, Woolf o Borges. En palabras de Rancière: me interesa la literatura "como principio de desclasificación de los discursos"27.

En diversos trabajos, como La parole muette. Essai sur les contradictions de la littérature (2010), Malaise dans l'esthétique (2004) o Politique de la littérature (2007), Rancière considera que los modos discursivos de la literatura se presentan como modos de disentimiento, que contrasta con otros modos de pensamiento que tienden al consenso, que Rancière denomina arte ético. Para Rancière, el consenso produce una ruptura en la base de la democracia, que es poder decir lo contrario, disentir. Este argumento parece recuperar un concepto clásico, el de parresía $^{28}$, que se

\footnotetext{
${ }^{25}$ Ibidem, p. 25.

${ }^{26}$ C. Menke, Die Souveränität der Kunst: Ästhetische Erfahrung nach Adorno und Derrida, Frankfurt am Main, Suhrkamp Verlag, 1991. (Trad. La soberanía del arte. La experiencia estética según Adorno y Derrida, Madrid, Visor, 1997).

${ }^{27} \mathrm{~J}$. Rancière, La palabra muda. Ensayo sobre las contradicciones de la literatura, Buenos Aires, Eterna Cadencia, 2009.

${ }^{28}$ Este proyecto se puede comprender desde la exclusión de la sinrazón por la razón moderna (instrumental). Para un desarrollo del concepto de parresía en relación con la literatura, puede verse mi trabajo: "Razón y Sinrazón en El licenciado Vidriera, El casamiento engañoso y El coloquio de los perros", en: Confluencia. Revista Hispánica de Cultura y Literatura. 28 - 2, Northern Colorado, University of Northern Colorado, 2013, pp. 180 - 195.
} 
define como "decir franco", o posibilidad de enfrentarse al decir común poniéndose en peligro uno mismo, que encontramos también en los últimos trabajos de M. Foucault asociado al origen de la democracia.

Asimismo, coincidiendo con propuestas cercanas a las de M. Foucault, la literatura ofrecería además formas inéditas de organización del espacio social. Y es precisamente desde la autonomía de la literatura desde donde es posible la creación de nuevos espacios comunitarios. Ciertamente, encontramos un precedente clásico en las reflexiones platónicas sobre la literatura pues es sabido que la condena de la literatura en la República platónica no proviene tanto del peligro de su capacidad representativa (mimesis), sino de, en una sociedad jerarquizada y determinada, hacer pensar al espectador que puede ser de otro modo. $\mathrm{O}$ de lo que en el proyecto foucaultiano se corresponde con la necesidad de crear formas inéditas de ser, o del carácter necesariamente creativo que ha de tener la filosofía como literatura ${ }^{29}$.

Por su parte, Nancy opina en el capítulo “¿Por qué hay varias artes y no una sola? de Las musas ${ }^{30}$ que: "El arte no tiene que ver con el "mundo" entendido como exterioridad simple, medio o naturaleza. Tiene que ver con el ser en el mundo en su propio surgimiento" 31 . La experiencia estética tendría para Nancy un carácter comunitario en tanto que es definida por el autor como "dotar de un sentido que compartimos"). En los capítulos que dedica a la noción de comunismo literario en la versión inglesa del segundo volumen de las Musas ${ }^{32}$ y en La comunidad desobrada ${ }^{33}$, J.-L., Nancy, afirmaba que lo que hay de político en la literatura, no es su representación de la sociedad, sino que parte de ella ayuda a cimentar el vínculo social ${ }^{34}$. Este vínculo no es una comunión (en el sentido de la catarsis comunitaria de la tragedia clásica, o del consenso que criticaba Rancière), está incompleta, es un nudo que preserva la separación, y que contiene también la posibilidad de desigualdad y dominación. La relación entre política y literatura sería disyuntiva o diferencial. Como J. Rancière, J.-L. Nancy considera que existe en esta relación algo esencial para la democracia: "una comunidad inconstante, suspendida entre la contingencia y la resolución de su acto", Contradicción o tensión irresoluble, y por lo tanto no dialéctica. De manera que, concluye Nancy, "la literatura es política en un sentido paradójico en tanto que une y separa en el mismo espacio" 35 .

\footnotetext{
${ }^{29}$ G. Deleuze, Qu'est -ce que la philosophie?, París, Minuit, 1999.

${ }^{30}$ J.-L. Nancy, Las musas, vol. I, Buenos Aires, Amorrortu, 2008.

${ }^{31}$ Ibidem, p. 31.

${ }^{32}$ J. -L. Nancy, Multiple Arts. The Muses II, París, Galilée, 2006.

${ }_{33}^{3}$ J. -L. Nancy, La communauté désouvrée, París, Galilée, 1999. (Trad. La comunidad desobrada, Madrid, Arena Libros, 2001).

${ }^{34}$ J. -L. Nancy, Multiple Arts. The Muses II, op. cit., p. 24.

${ }^{35}$ Ibidem., p. 26.
} 
Finalmente, en Christoph $\mathrm{Menke}^{36}$, la experiencia estética sería también genuinamente comunitaria, pero en tanto que es previa al gusto individual, pues el gusto se forma también en el proceso de la educación que es siempre social (Bildung). Para Menke es la fuerza (Kraft) que se desarrolla en la educación (como desarrollo de la potencialidad) la que nos permite transgredir estéticamente nuestra existencia social, en la que experimentamos que somos iguales (la igualdad o la libertad serían características sociales, comunitarias, antes que naturales). La igualdad política, pues, sería un efecto estético. Concluye C. Menke en el texto que presentó en la Documenta de 2011, "Äesthetik der Gleichheit" ${ }^{37}$ : "Nos hacemos a nosotros estéticamente iguales; estéticamente, nos hacemos a nosotros mismos iguales". Esta propuesta contrasta la propuesta individualista de la experiencia estética que defiende en sus trabajos Peter Sloterdijk. Para Sloterdijk, el imperativo categórico es una ley de la razón, pero la experiencia estética no obedecería a leyes universales, ni comunitarias: no existe el imperativo estético. Como afirma Schinckel en In Media Res: Peter Sloterdijk's Spheorological Poetics of being ${ }^{38}$, su estética se defiende desde una dimensión ética individual.

Concluimos de este modo esta breve reflexión que se propone como una apertura del diálogo entre la estética política y la teoría de la literatura, entre la teoría de la cultura y la teoría del texto. Este camino permitiría, como se ha descrito, comprender la obra literaria en toda su plurivocidad, no sólo atendiendo a su temática, sino que el estudio de la forma en este nuevo contexto de la teoría de la cultura, nos permitiría estudiar cómo las condiciones formales de la obra literaria se enfrentan a las instituciones de poder. La teoría de la literatura y la estética de la literatura están sujetas a los objetos históricos del arte, a su pluralidad, y a sus transformaciones. De este modo, la teoría de la literatura encontraría de nuevo su lugar, un formalismo histórico capaz de leer todas las dimensiones de las obras literarias: estética, formal y política. Y sin duda, las propuestas de estos autores, R. Rorty, J. Rancière, C. Menke y J.-L. Nancy, entre otras, se presentan como modelos de lectura surgidos de este fructífero diálogo.

\footnotetext{
${ }^{36}$ C. Menke, Kraft. Ein Grundbegriff äesthetischer Anthropologie, Frankfurt a. M, Suhrkamp, 2008.

${ }^{37}$ C. Menke, Aesthetics of Equality/ Äesthetik der Gleichheit, Documenta (13), Kassel, Hatje Cantz Verlag, 2011, p. 16.

${ }^{38}$ W. Schinkel, In Medias Res: Peter Sloterdijk's Spherological Poetics of Being, Amsterdam, Amsterdam University Press, 2011.
} 